Marquette University

e-Publications@Marquette

9-1-2003

Effect of Computer Keyboard Slope on Wrist Position and Forearm Electromyography of Typists Without Musculoskeletal Disorders

Guy G. Simoneau

Marquette University, guy.simoneau@marquette.edu

Richard W. Marklin

Marquette University, richard.marklin@marquette.edu

Joseph E. Berman

Marquette University, joseph.berman@marquette.edu

Published version. Physical Therapy, Vol. 83, No. 9 (2003): 816-830. DOI. (C) 2003 American

Physical Therapy Association. Used with permission. 


\section{Effect of Computer Keyboard Slope on Wrist Position and Forearm Electromyography of Typists Without Musculoskeletal Disorders}

Background and Purpose. Positioning a computer keyboard with a downward slope reduces wrist extension needed to use the keyboard and has been shown to decrease pressure in the carpal tunnel. However, whether a downward slope of the keyboard reduces electromyographic (EMG) activity of the forearm muscles, in particular the wrist extensors, is not known. Subjects and Methods. Sixteen experienced typists participated in this study and typed on a conventional keyboard that was placed on slopes at angles of 7.5, $0,-7.5$, and -15 degrees. Electromyographic activity of the extensor carpi ulnaris (ECU), flexor carpi ulnaris (FCU), and flexor carpi radialis (FCR) muscles was measured with surface electrodes, while the extension and ulnar deviation angles of the right and left wrists were measured with electrogoniometers. Results. Wrist extension angle decreased from approximately 12 degrees of extension while typing on a keyboard with a 7.5-degree slope to 3 degrees of flexion with the keyboard at a slope of -15 degrees. Although the differences were in the range of $1 \%$ to $3 \%$ of maximum voluntary contraction (MVC), amplitude probability distribution function (APDF) of root-mean-square EMG data points from the ECU, FCU, and FCR muscles varied across keyboard slopes. Discussion and Conclusion. Wrist extension decreased as the keyboard slope decreased. Furthermore, a slight decrease in percentage of MVC of the ECU muscle was noted as the keyboard slope decreased. Based on biomechanical modeling and published work on carpal tunnel pressure, both of these findings appear to be positive with respect to comfort and fatigue, but the exact consequences of these findings on the reduction or prevention of injuries have yet to be determined. The results may aid physical therapists and ergonomists in their evaluations of computer keyboard workstations and in making recommendations for interventions with regard to keyboard slope angle. [Simoneau GG, Marklin RW, Berman JE. Effect of computer keyboard slope on wrist position and forearm electromyography of typists without musculoskeletal disorders. Phys Ther. 2003;83:816-830.]

Key Words: Computer keyboard, Slope angle, Typing, Wrist angle, Wrist extension. 


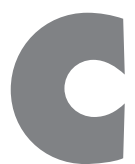

omputers are ubiquitous in our society, with 100 million computers estimated to be in use in the United States in 2000. ${ }^{1}$ Except for rare cases, every computer has a keyboard for text and data entry. The occupational risk factors of musculoskeletal disorders (MSDs), such as carpal tunnel syndrome and tenosynovitis, are thought to be due to excessive use (up to 100,000 keystrokes per $\left(\mathrm{day}^{2}\right)$ and due to the use of deviated wrist postures. ${ }^{3,4}$ An extensive review ${ }^{1}$ of the literature on the association between keyboard usage and prevalence of MSDs showed that the prevalence of keyboard-related MSDs among computer users, based on symptoms or physical examination findings, ranged from $9 \%$ to $50 \%$, as compared with $4.5 \%$ to $17 \%$ among reference groups who were exposed to low levels of or no keyboard work. Compared with findings for a reference group, the odds ratios for keyboard-related MSDs among computer users were from 0.5 to 9.9 for the neck and shoulders and from 0.7 to 10.1 for the hand, wrist, or elbow. ${ }^{1}$ Although most of the studies reviewed ${ }^{1}$ had limitations of comparisons across groups at a single time period and were based on self-reported health measures, the odds ratios and prevalence of upper-extremity MSDs of computer users, as compared with findings for a reference group, suggest that computer keyboards may contribute to MSDs affecting the upper extremities.

Only a few researchers have investigated the magnitude of forearm muscle electromyographic (EMG) activity during typing on computer keyboards. Electromyography was used to measure the muscle activity of the flexor digitorum superficialis (FDS) and extensor digitorum communis (EDC) muscles while subjects typed on keyboards with keys of varying stiffness. ${ }^{5}$ These researchers used an amplitude probability distribution function (APDF) for analyzing EMG data, which is a method to quantify the level of EMG activity for a task in which the muscles are changing in length. These researchers found that the 50th percentile of the EMG signals for the FDS muscle was approximately $7 \%$ of maximum voluntary contraction (MVC), which means that $50 \%$ of the root-mean-square (RMS) EMG data points were under

GG Simoneau, PT, PhD, ATC, is Associate Professor, Department of Physical Therapy, Marquette University, Milwaukee, Wis.

RW Marklin, PhD, CPE, is Associate Professor, Department of Mechanical and Industrial Engineering, Marquette University, PO Box 1881, Milwaukee, WI 53201-1881 (USA) (richard.marklin@marquette.edu). Address all correspondence to Dr Marklin.

JE Berman, PT, MHS, ATC, is Research Assistant, Department of Physical Therapy, Marquette University.

Dr Simoneau and Dr Marklin provided concept/idea/research design, writing, facilities/equipment, and consultation (including review of manuscript before submission). Mr Berman provided data collection, and Dr Simoneau and Mr Berman provided data analysis, subjects, and institutional liaisons. Dr Simoneau provided project management and fund procurement. The authors acknowledge The Arthritis Foundation and the Office Ergonomics Research Committee (OERC) for partially funding this project. In addition, the authors thank Kevin Garrison, John Monroe, Sarah Welsh, Dr Paula Papanek (Marquette University), and Dr Roberta Henderson (Finch University of Health Sciences, Chicago, Ill) for their technical assistance.

Testing of human subjects was approved by the Marquette University Office of Research and Sponsored Program's Institutional Review Board (HR-94-292).

This research was presented in partial and condensed form at the International Society of Biomechanics Conference; Zurich, Switzerland; July 8-13, 2001. 


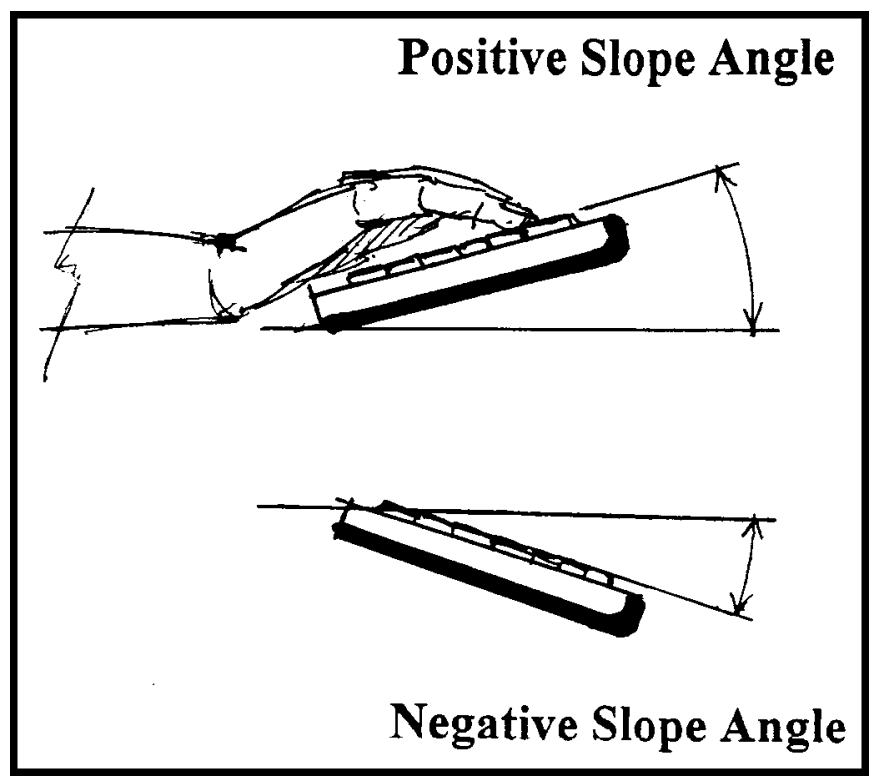

Figure 1.

Computer keyboards illustrating what were considered positive and negative slopes in the study.

7\% MVC. The 10th percentile (considered a measure of the baseline of muscle activity) was about $2 \%$ MVC, which means that $10 \%$ of the RMS EMG data points were under $2 \%$ MVC during typing trials or that muscle activity level was greater than $2 \%$ MVC for $90 \%$ of the typing trial. The corresponding 50th and 10 th percentiles for the EDC muscle (11.5\% and 6.5\% MVC, respectively) were much greater than for the FDS muscle. These results ${ }^{5}$ are similar to findings from other researchers ${ }^{6}$ who measured median (50th percentile) EMG activity from the EDC muscle that ranged from $6.5 \%$ to $14 \% \mathrm{MVC}$ while subjects were typing on various keyboards. The greater activity of the dorsal forearm musculature was most likely due to the postural requirement of holding the hand and fingers above the keyboard while fingers were typing keys. ${ }^{5}$

Modifications to the design of the keyboard could possibly reduce the magnitude of forearm muscle activity. A change to the keyboard that might decrease muscle activity of extensor forearm muscles is sloping the keyboard downward. As illustrated in Figure 1, keyboard slope is the angle of the plane of keytops to a horizontal. A typical conventional keyboard has a built-in slope of about 6 degrees. We have shown that changing the slope of the keyboard in a downward direction can change wrist extension angle and not impair typing speed and accuracy. ${ }^{7}$ Wrist extension angle decreased 1 degree for every 2-degree decrease in keyboard slope angle as the keyboard was positioned at 15 to -15 degrees of slope. Mean wrist extension angle decreased to less than 15 degrees when the keyboard was positioned with a slope of 0 degrees or lower. In theory, wrist extension angles close to the anatomical neutral position (compared with large wrist extension angles) result in less risk of distal upper-extremity MSDs because the pressure in the carpal tunnel is lower and forces pressing against the median nerve and flexor tendons are less. ${ }^{8-10}$

We know of no published studies in which the effect of computer keyboard slope on EMG activity levels of the forearm musculature was examined. There are a number of physiological and biomechanical factors that could affect the amount of activity of the forearm extensors and flexors as a keyboard slope is changed. As the wrist flexion/extension angle changes with the change in keyboard slope, some of those factors include: a change in muscle length, ${ }^{11}$ a change in the muscles' moment arm at the wrist joint, ${ }^{12}$ and a change in the location of the hand's center of mass (which exerts a passive moment at the wrist). The EMG activity of the forearm musculature could provide some insight into how keyboard slope affects the recruitment level of the wrist extensors and flexors.

The objective of this study was to determine the effect of computer keyboard slope angle on forearm musculature EMG activity in individuals without any upper-extremity symptoms of MSDs. All subjects were 10-digit "touch" typists. We hypothesized that percentage of MVC of the extensor carpi ulnaris (ECU) muscle would change as keyboard slope decreased. If there is a difference, changing the slope of the keyboard in a downward direction may be an intervention that could be used in the design of new computer workstations and modifications to existing workstations.

\section{Method}

\section{Subjects}

Fifteen women and 1 man (mean age $=42.5$ years, $\mathrm{SD}=8.7$, range $=27-53$ ) participated in the study. The number of subjects was determined a priori based on statistical power analysis to ensure type I error did not exceed 0.05 and type II error did not exceed 0.20 . This analysis indicated that a minimum of 15 subjects was necessary in a repeated-measures design of one independent variable (keyboard slope angle) with 4 levels (slope angles) to detect a difference of $5 \%$ MVC for 50th percentile APDF of forearm RMS EMG activity between pairs of keyboard slopes. We speculated a priori that $5 \%$ MVC is clearly a meaningful effect size. A standard deviation of 4\% MVC was assumed for each slope angle. A similar power analysis, which resulted in a minimum of 15 subjects, was performed for detecting a 5-degree difference in wrist extension angle between pairs of keyboard slopes and assuming a standard deviation of 4 degrees within each keyboard slope. 


\section{Please rate the overall ease of use of this keyboard}

\begin{tabular}{|c|c|c|c|c|c|}
\hline $\begin{array}{c}\text { Very difficult } \\
\text { to use }\end{array}$ & $\begin{array}{c}\text { Somewhat } \\
\text { difficult to } \\
\text { use }\end{array}$ & $\begin{array}{c}\text { Difficult to } \\
\text { use }\end{array}$ & $\begin{array}{c}\text { Slightly easy } \\
\text { to use }\end{array}$ & $\begin{array}{c}\text { Somewhat } \\
\text { easy to use }\end{array}$ & $\begin{array}{c}\text { Very easy to } \\
\text { use }\end{array}$ \\
1 & 2 & 3 & 4 & 5 & 6 \\
\hline
\end{tabular}

2. Please rate your overall comfort while using this keyboard

\begin{tabular}{|c|c|c|c|c|c|}
\hline $\begin{array}{c}\text { Very } \\
\text { uncomfortable }\end{array}$ & $\begin{array}{c}\text { Somewhat } \\
\text { uncomfortable }\end{array}$ & $\begin{array}{c}\text { Slightly } \\
\text { uncomfortable }\end{array}$ & $\begin{array}{c}\text { Slightly } \\
\text { comfortable }\end{array}$ & $\begin{array}{c}\text { Somewhat } \\
\text { comfortable }\end{array}$ & $\begin{array}{c}\text { Very } \\
\text { comfortable }\end{array}$ \\
1 & 2 & 3 & 4 & 5 & 6 \\
\hline
\end{tabular}

Figure 2.

Questionnaire used to assess ease of use and overall comfort of each keyboard slope angle.
Tinel tests have reported sensitivities of $71 \%$ and $44 \%$, respectively, and specificities of $80 \%$ and $94 \%$, respectively. ${ }^{13,14}$ All subjects gave informed consent prior to participation in the study.

\section{Experimental Design}

A repeated-measures experimental design was used to determine wrist angle position and percentage of MVC for EMG activity of the forearm musculature from subjects typing on a conventional keyboard with the slope at 4 angles $\left(7.5^{\circ}, 0^{\circ},-7.5^{\circ}\right.$, and $\left.-15^{\circ}\right)$. During the testing session, all subjects typed on all 4 slopes. The order of use was presented randomly for each subject.
All subjects typed at least 40 words per minute (wpm) using the 10-digit "touch" method (capable of typing accurately without looking at the keys) and worked in jobs requiring typing at least 2 hours per workday. The subjects were clerical workers recruited from serviceoriented businesses within the metropolitan Milwaukee area. Typing speed was confirmed by Typing Tutor 6.0 software* during a short typing practice session before the commencement of data collection. The subjects' mean shoulder width was $38.1 \mathrm{~cm}$ $(\mathrm{SD}=2.2$, range $=33.7-42.9)$. The mean length of the right forearm and hand was $44.3 \mathrm{~cm}(\mathrm{SD}=2.5$, range $=39.9-48.4)$, and that of the left forearm and hand was $44.3 \mathrm{~cm}(\mathrm{SD}=2.7$, range $=39.6-48)$. A person's shoulder width and the length of the forearm and hand are important because they can affect the ulnar deviation angle of the wrist while the person types.

At the time subjects were recruited, the subjects were asked if they were free of pain or discomfort related to typing. Based on answers to questions regarding various body segments, subjects indicated they were free of symptoms related to musculoskeletal injury, pain, and discomfort that could interfere with typing. Immediately prior to testing, the subjects were asked questions about pain, tingling, and numbness in their upper extremities. This was done to further ensure that the subjects were free of medical problems that could interfere with typing and to confirm that they did not have symptoms in their distal upper extremities that were related to typing. Furthermore, all subjects tested negatively for Phalen and Tinel tests for carpal tunnel syndrome. Phalen and

* Kriya Systems Inc, Sterling, Va.

\section{Dependent Variables}

The dependent variables for this study were the following:

1. 10th, 50th, and 90th percentiles of the APDF of the RMS EMG signal expressed as a percentage of MVC from the right and left ECU, flexor carpi radialis (FCR), and flexor carpi ulnaris (FCU) muscles. The ECU, FCR, and FCU muscles were selected because these muscles can be reliably monitored with surface EMG during typing. ${ }^{15-17}$ The use of the APDF is a widely accepted method of quantifying EMG signals from forearm muscles during typing tasks. ${ }^{8}$

2. Mean, maximum, and minimum wrist extension and ulnar deviation angles of the right and left wrists.

3. Typing speed and accuracy. Typing speed for each keyboard slope was measured in words per minute for a duration of 6 minutes. Typing accuracy, also measured over a 6-minute period, was defined as the difference between the total number of characters typed and the total number of errors left in the document divided by the total number of characters.

4. Psychophysical assessment of ease of use and comfort level. For each keyboard slope, the subjects rated the keyboard's overall comfort and ease of use on a 1 to 6 scale, as shown in Figure 2.

\section{Apparatus}

A fixture for a conventional QWERTY keyboard was built so the keyboard could be positioned with slopes at positive and negative angles to the horizontal. As shown in Figure 3, adjustable-length stilts made out of threaded bolts adjusted the keyboard's slope to 4 positions: 7.5, 0 , -7.5 , and -15 degrees. A wrist rest, which was built so it was on the same plane as the keyboard, was used in an 


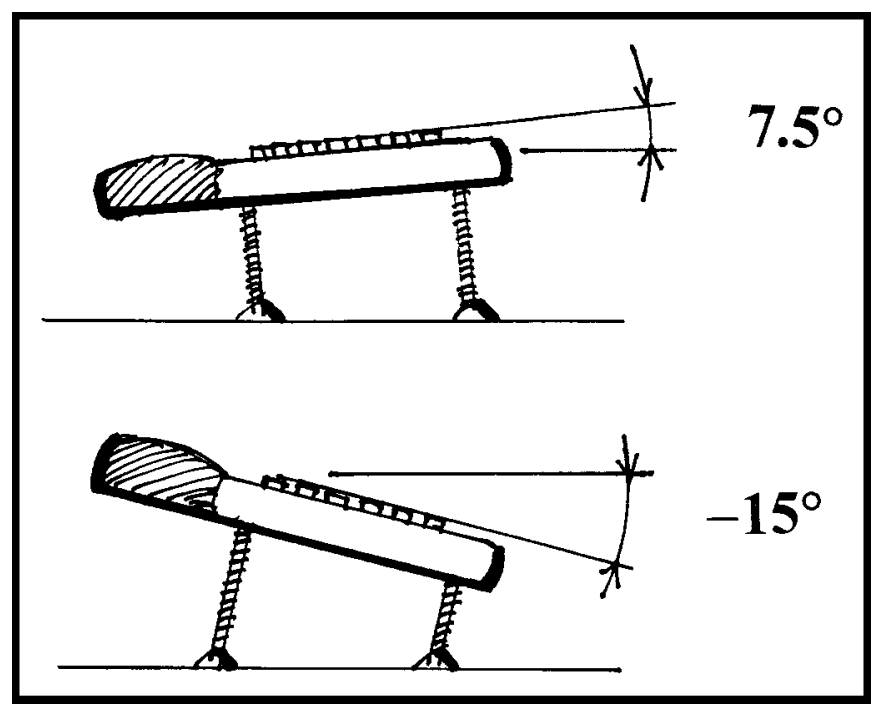

Figure 3.

Fixture for varying the slope of the test keyboard. The shaded object on the left is the built-in wrist rest.

effort to control the relative height of the wrist in relationship to elbow height. The wrist rest was not used to support the wrist while typing. An adjustable computer workstation (desk and chair) was used for testing, and adjustments of chair and keyboard height were made as described in the "Procedure" section. Typing speed and accuracy were measured with the Typing Tutor 6.0 software. Biaxial electrogoniometers ${ }^{\dagger}$ attached to the dorsum of the wrist measured wrist extension and ulnar deviation angles while typing. These electrogoniometers, which are of strain gauge type and are lightweight and unobtrusive to the typist, measured wrist angles in the flexion/extension and radial/ulnar planes simultaneously. The accuracy of the goniometers in each plane was 2 degrees. As assessed by intraclass correlation coefficient (ICC) 18,19 on our sample of 16 subjects, intertrial reliability for the measurement of wrist extension and ulnar deviation angles was in excess of .90 . These reliability values are of similar magnitude to those previously reported. ${ }^{7}$

The RMS EMG data for the left and right ECU, FCU, and FCR muscles were collected with surface electrodes and EMG system from Therapeutics Unlimited. ${ }^{\ddagger}$ The pairs of surface electrodes contained circuitry for preamplification with a gain of 35 and minimized artifact. ${ }^{20}$ Raw bipolar EMG data from the electrodes were processed utilizing the RMS method, which produced a linear envelope or average EMG voltage over the data collection period..$^{21}$ The time-constant window over which the RMS data were calculated was 55 milliseconds. Online wrist joint position from the electrogoniometers and

\footnotetext{
${ }^{\dagger}$ Biometrics Corp, PO Box 340, Ladysmith, VA 22501.

‡ Therapeutics Unlimited, 2835 Friendship St, Iowa City, IA 52240 .
}

EMG RMS data were sampled at $300 \mathrm{~Hz}$ and fed into a 12-bit analog-to-digital converter ${ }^{\S}$ and stored on a personal computer operated with custom-written LabVIEW software. $\$$ Based on 5 samples of 30 seconds of EMG data collected over a 6-minute typing session, intertrial ICCs of the 50th percentile of the APDF of the RMS EMG data were $.99, .94$, and .98 for the left ECU, FCU, and FCR muscles, respectively. These ICCs were calculated from all 16 subjects when the keyboard was positioned at a slope of 7.5 degrees. The ICC data were similar for muscles of the right forearm.

Fifteen seconds of normalized EMG data for the right ECU muscle expressed as percentage of MVC is shown in Figure 4. Data were collected with the keyboard positioned at a slope of 7.5 degrees.

\section{Procedure}

On arrival at the laboratory, each subject signed an approved human consent form. A brief medical survey questionnaire was completed, and anthropometric dimensions of the trunk, arms, forearms, and hands along with range of motion of the wrists were recorded. The electrogoniometers and surface EMG electrodes were attached to the subject's left and right wrists and forearms. After the subject's wrists were placed in position, the EMG electrodes were located according to widely accepted guidelines. ${ }^{22} \mathrm{~A}$ muscle contraction was detected by palpation. The electrogoniometers were then calibrated with each subject's wrist resting on a custom-built calibration table in which the wrist was placed in an anatomically neutral $\left(0^{\circ}\right)$ position with respect to flexion/extension and radial/ulnar deviation. One second of calibration data were collected in this position. A standard degree/volt conversion factor was used to obtain the wrist joint's angular position as published by the manufacturer and verified in our laboratory.

Maximum voluntary contraction and resting EMG voltages for each of the 3 muscles tested for each forearm were recorded with the wrists extended 8 degrees and ulnarly deviated 10 degrees for the right side and 15 degrees for the left side. These are the wrist positions commonly assumed by typists when typing on a conventional keyboard. ${ }^{4}$ For the measurement of MVC, a custom-built fixture was used, as shown in Figure 5. For each muscle, the subject was instructed on the specific movement to perform (eg, wrist extension and ulnar deviation for the ECU muscle). The subject performed several practice trials. The subject then made 3 contractions of 5 seconds' duration for which data were saved. For each trial, an automated moving window identified the 0.5 -second period where the maximum RMS EMG 


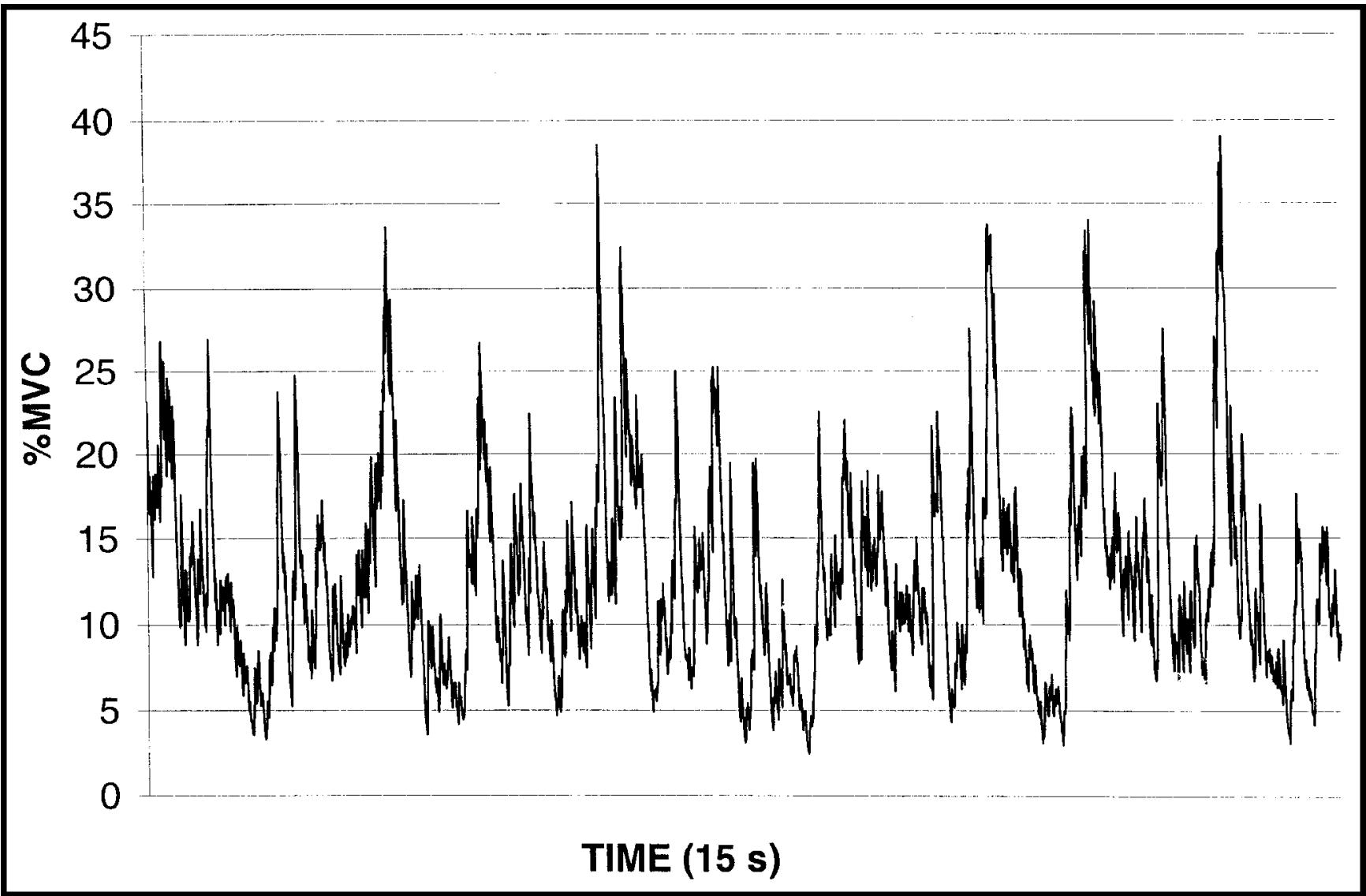

Figure 4.

Fifteen seconds of normalized root-mean-square electromyographic data of the right extensor carpi ulnaris muscle expressed as a percentage of maximum voluntary contraction. The keyboard was at a positive slope of 7.5 degrees.

signal was measured. We used the maximum EMG value of the 3 contractions for normalization (ie, obtaining a percentage of MVC). The ICCs for percentage of MVC for the left ECU, FCU, and FCR muscles were .98, .96, and .98 , respectively. The ICCs for percentage of MVC for the right ECU, FCU, and FCR muscles were .99, .98, and .96, respectively. After all of the electrodes and goniometers were attached, the subject then sat on a height-adjustable chair seat next to a video-display terminal (VDT) workstation that was set up according to widely accepted guidelines for VDT workstations. ${ }^{23}$ For all 4 slopes of the keyboard, the height of the chair seat was adjusted so that the subject's right ulnar styloid process was level with the lateral epicondyle while typing.

The keyboard was adjusted to the first of the 4 randomly assigned slopes, and each subject practiced typing at that keyboard setting for 3 minutes. Then the subject typed for 6 minutes. Wrist position was recorded and EMG data were collected during five 30-second intervals throughout the 6-minute typing session. The subject then rested for 5 minutes while the keyboard was adjusted to the next slope. The subject practiced typing for 3 minutes with the new slope, and then for another 6 minutes while data were collected. This procedure was repeated until the subject typed on the keyboard set at each of the 4 angles. ${ }^{24}$

\section{Data Processing and Statistical Analysis}

Wrist angle data. After the wrist extension and ulnar deviation voltage data were converted to angular measurements, the angular data were filtered by a secondorder, double-pass Butterworth filter with a cutoff frequency of $7 \mathrm{~Hz}$. For each slope, the mean, maximum, and minimum wrist extension and ulnar deviation angles of the subjects were computed for each of the five 30 -second trials. The summary statistics for the 5 trials within each slope of the keyboard were then averaged for each subject. The mean, minimum, and maximum wrist angle data for wrist extension and ulnar deviation were analyzed with a 2-way analysis of variance (ANOVA) for repeated measures followed by a Tukey honestly significant difference (HSD) multiple-comparison post hoc test when main effects and any interactions were present. The 2 independent variables were hand (2 levels: right and left) and keyboard slope (4 levels: $7.5^{\circ}$, $0^{\circ},-7.5^{\circ}$, and $\left.-15^{\circ}\right)$. Statistical significance was set at $P<.05$. 


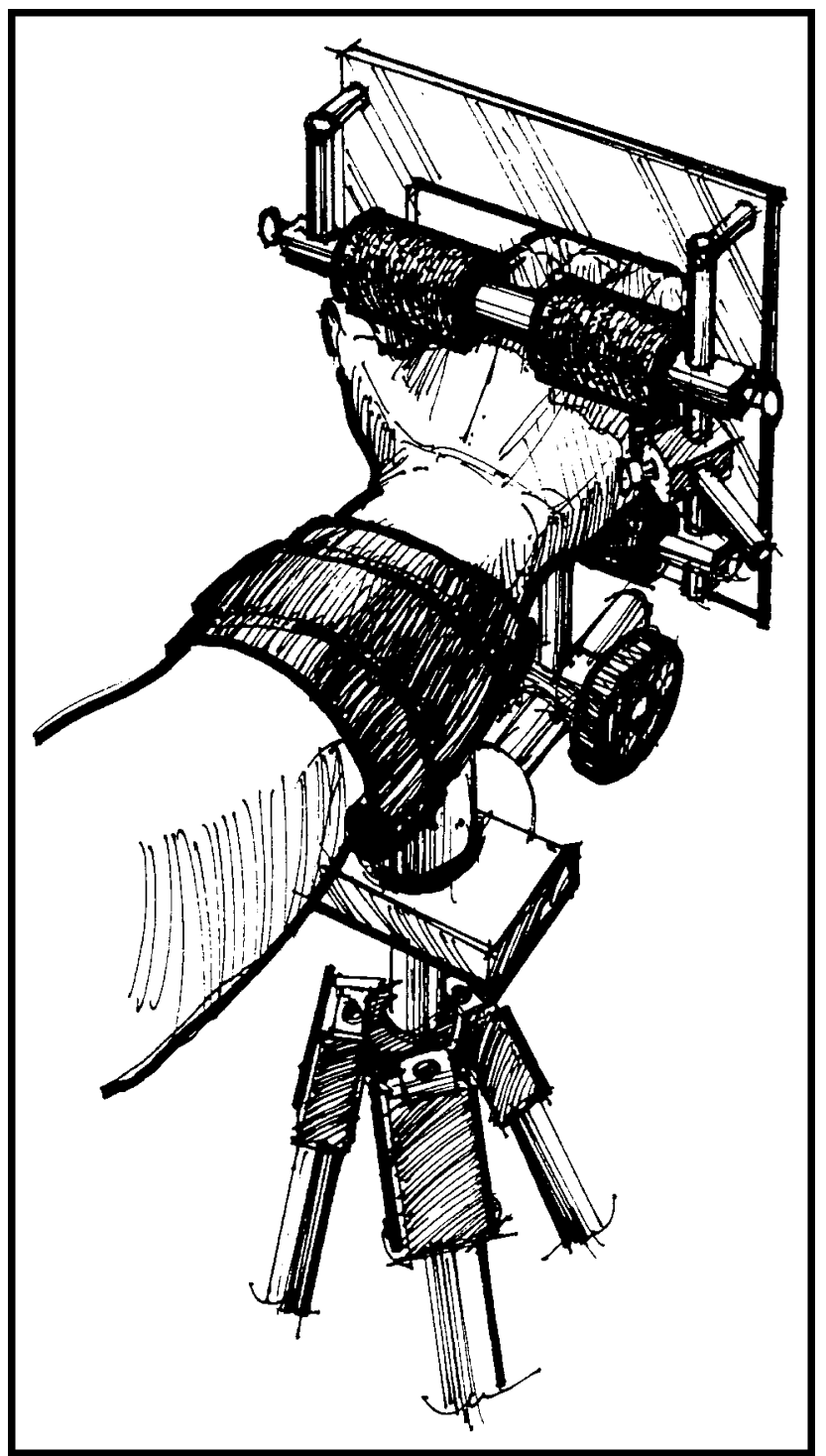

Figure 5 .

Custom-built fixture to allow subjects to make maximum voluntary contractions (MVCs) so that maximal electromyographic voltages $(100 \% \mathrm{MVC})$ of the extensor carpi ulnaris, flexor carpi ulnaris, and flexor carpi radialis muscles could be monitored.

EMG data. The EMG data during typing sessions were normalized to a percentage of MVC, which is the widely accepted method for analyzing EMG data. ${ }^{8,9}$ In post hoc processing of each of the 5 trials of EMG data per slope, the 10th, 50th, and 90th percentile APDFs of the RMS EMG data were calculated with LabVIEW software. For each muscle, the APDF summary statistics were then averaged over the 5 trials for each keyboard slope. Similar to wrist position data, the 10th, 50th, and 90th percentile APDFs of the RMS EMG data for the ECU, FCU, and FCR muscles were analyzed individually with a 2-way ANOVA for repeated measures, followed by a Tukey HSD multiple-comparison post hoc test when main effects and any interactions were present. The 2 independent variables were hand (2 levels: right and left) and keyboard slope (4 levels: $7.5^{\circ}, 0^{\circ},-7.5^{\circ}$, and $-15^{\circ}$ ) Statistical significance was set at $P<.05$.

Typing performance data. Typing speed and accuracy were averaged in the same manner as wrist angle and EMG data and were analyzed with a one-way ANOVA for repeated measures (one independent variable: keyboard slope; 4 levels: $7.5^{\circ}, 0^{\circ},-7.5^{\circ}$, and $\left.-15^{\circ}\right)$. Statistical significance was set at $P<.05$.

Psychometric data. Ease-of-use and comfort data collected after subjects typed on each keyboard slope angle were analyzed using a Friedman ANOVA by ranks to determine if there was a difference between groups overall and a Wilcoxon signed rank test to determine differences between pairs of keyboard slopes. Statistical significance was set at $P<.05$.

\section{Results}

\section{Wrist Position}

Wrist extension. Mean wrist extension angles decreased as the keyboard slope decreased, as indicated in Table 1 and shown in Figure 6. Subjects extended their wrists approximately 12 degrees when the keyboard was positioned at a slope of 7.5 degrees and moved their wrists to a mean flexion angle of approximately 3 degrees at the slope of -15 degrees. Extension angles did not vary between the right and left wrists, and there was no interaction between keyboard slope and hand.

Ulnar deviation. Keyboard slope angle had a main effect on mean ulnar deviation in that ulnar deviation increased 3 to 5 degrees as the keyboard was sloped from 7.5 degrees to -15 degrees (an increase in mean ulnar deviation from $12.7^{\circ}$ to $15.7^{\circ}$ for the left wrist and from $9.1^{\circ}$ to $14.9^{\circ}$ for the right wrist), as indicated in Table 2 . The varying increases in ulnar deviation between the left and right wrists as the keyboard slope was changed $\left(3^{\circ}\right.$ for the left wrist and $5^{\circ}$ for the right wrist) resulted in an interaction between keyboard slope and hand. There was no main effect for hand on mean wrist ulnar deviation. A summary of differences across keyboard slopes for the mean, maximum, and minimum ulnar deviation values is presented in Table 2 .

\section{Forearm EMG Activity}

ECU muscle. The ECU muscle's percentage of MVC did not vary between left and right wrists for the 10th, 50th, and 90th percentile APDF levels, which were approximately $6 \%, 12 \%$, and 25\% MVC (Tabs. 3-5). For all 3 APDF levels, the ECU muscle's percentage of MVC decreased when the keyboard was moved downward in slope. As shown in Table 4 and depicted in Figure 7, the 
Table 1.

Average, Standard Deviation, and Minimum and Maximum Values for the Mean, Maximum, and Minimum Wrist Extension Angles (in Degrees) of Individuals ( $N=16)$ Typing on a Keyboard With Slopes at 4 Angles $^{a}$

\begin{tabular}{|c|c|c|c|c|c|c|c|c|}
\hline & \multicolumn{2}{|c|}{$7.5^{\circ}$ Slope } & \multicolumn{2}{|c|}{$0^{\circ}$ Slope } & \multicolumn{2}{|c|}{$-7.5^{\circ}$ Slope } & \multicolumn{2}{|c|}{$-15^{\circ}$ Slope } \\
\hline & Left & Right & Left & Right & Left & Right & Left & Right \\
\hline \multicolumn{9}{|c|}{ Mean wrist extension } \\
\hline SD & 7.9 & 6.9 & 7.8 & 6.6 & 7.1 & 5.8 & 7.1 & 6.1 \\
\hline Minimum & -6.3 & -5.7 & -6.9 & -7.7 & -11.2 & -11.7 & -13.2 & -15.5 \\
\hline Maximum & 28.0 & 25.0 & 25.9 & 19.9 & 16.6 & 14.0 & 14.2 & 10.1 \\
\hline \multicolumn{9}{|c|}{ Maximum wrist extension } \\
\hline Average & $24.2^{b}$ & 23.7 & $20.1^{b}$ & 20.0 & $14.3^{b}$ & 15.2 & $9.3^{b}$ & 10.7 \\
\hline $\mathrm{SD}$ & 9.3 & 7.9 & 9.5 & 8.2 & 9.0 & 7.8 & 8.8 & 7.8 \\
\hline Minimum & 3.1 & 3.6 & 4.0 & 2.4 & 0.4 & -2.1 & -3.4 & -7.0 \\
\hline Maximum & 40.6 & 41.8 & 40.6 & 35.4 & 32.6 & 32.4 & 26.9 & 26.7 \\
\hline \multicolumn{9}{|c|}{ Minimum wrist extension } \\
\hline Average & $-1.7^{b}$ & -3.0 & $-5.8^{b}$ & -8.1 & $-12.5^{b}$ & -12.0 & $-15.9^{b}$ & -16.6 \\
\hline
\end{tabular}

${ }^{a}$ Wrist flexion angles are expressed as negative values. No differences existed between right and left wrists $(P>.05)$.

${ }^{b}$ Average of left and right wrist extension angles different from other slopes marked with same designation at the $P<.05$ level. Statistical analysis consisted of a one-factor repeated-measures analysis of variance $\left(\mathrm{F}_{3,45}\right.$ for keyboard slope main effect) and a Tukey honestly significant difference multiple-comparison post hoc test. Results indicate that for each keyboard slope, the wrist angle was different than for the other 3 slopes.

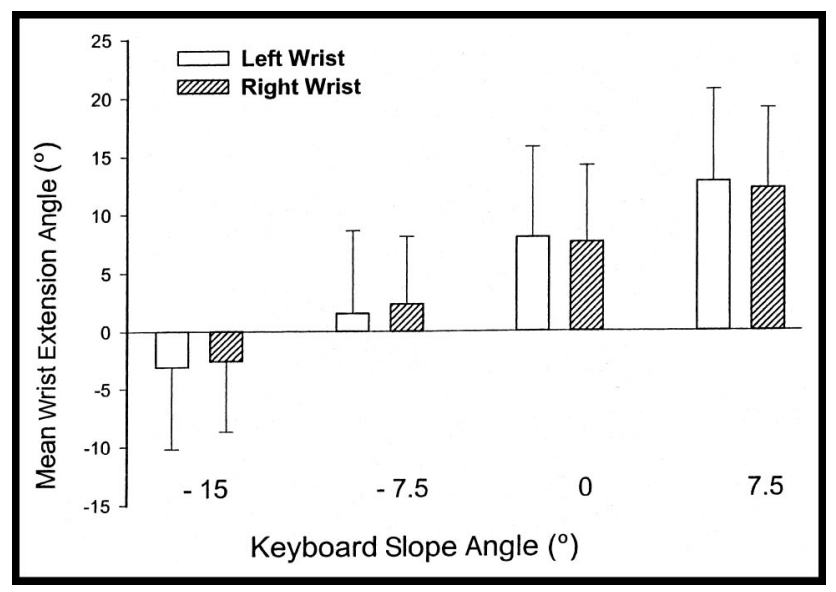

Figure 6.

Mean wrist extension angle as a function of keyboard slope angle and hand. Error bars represent one standard deviation.

mean 50th percentile APDF for the ECU muscle decreased from approximately $13.5 \%$ MVC to $11.5 \%$ MVC as the keyboard slope was changed from 7.5 to -15 degrees. Mean 10th and 90th percentile APDFs for the ECU muscles decreased in a similar manner as the 50th percentile APDF (Tabs. 3 and 5). There were no interactions between hand and keyboard slope. The differences across the 4 slopes of the keyboard are summarized in Tables 3 through 5 .

FCU and FCR muscles. Across the 10th, 50th, and 90th percentile APDFs, the magnitude of the percentage of MVC of the wrist flexors was lower than for their extensor counterpart, as indicated in Tables 3 through 5. At the 10th percentile APDF, the percentages of MVC of the FCU and FCR muscles were approximately $1 \%$ (Tab. 3), but then increased at different rates at higher APDF percentiles. The 50th percentile APDF was $5 \%$ to $8 \%$ MVC for the FCU muscle, whereas it was $2 \%$ to $4 \%$ MVC for the FCR muscle (Tab. 4). At the 90th percentile APDF, the difference was magnified in that the percentage of MVC ranged from $19 \%$ to $28 \%$ MVC for the FCU muscle and from $10 \%$ to $14 \%$ MVC for the FCR muscle (Tab. 5).

For the 10th percentile APDF for the FCU and FCR muscles, no main effects were found between hands and across slopes. In addition, no interactions were found. For the 50th percentile APDF for the FCU muscle, a small $(1 \%)$ increase in muscle activity was seen as the angle of the slope decreased. No difference existed between hands, and no interactions existed. For the 90th percentile APDF for the FCU muscle, a main effect was found between hands, and a main effect was also found for the angle of the slope. No interaction was found. For the 50th and 90th percentile APDFs for the FCR muscle, small differences (about 2\% and 4\%, respectively) existed between hands. There were no differences across slopes of the keyboards, and no interaction was found between the 2 factors. The differences across the 4 slope conditions are summarized in Tables 3 through 5. 
Table 2.

Average, Standard Deviation, and Minimum and Maximum Values of the Mean, Maximum, and Minimum Wrist Ulnar Deviation (in Degrees) of Individuals $(\mathrm{N}=16)$ Typing on a Keyboard With Slopes at 4 Angles ${ }^{a}$

\begin{tabular}{|c|c|c|c|c|c|c|c|c|}
\hline & \multicolumn{2}{|l|}{$7.5^{\circ}$ Slope } & \multicolumn{2}{|c|}{$0^{\circ}$ Slope } & \multicolumn{2}{|c|}{$-7.5^{\circ}$ Slope } & \multicolumn{2}{|c|}{$-15^{\circ}$ Slope } \\
\hline & Left & Right & Left & Right & Left & Right & Left & Right \\
\hline \multicolumn{9}{|c|}{ Mean ulnar deviation } \\
\hline SD & 5.6 & 6.6 & 5.1 & 6.9 & 5.2 & 6.8 & 5.3 & 6.9 \\
\hline Minimum & 2.3 & -4.0 & 5.3 & 0.0 & 5.2 & 3.1 & 5.5 & 3.6 \\
\hline Maximum & 21.6 & 16.6 & 23.0 & 22.6 & 22.7 & 23.0 & 22.7 & 25.2 \\
\hline Average & $20.0^{b, c, d, e}$ & $23.0^{f, g}$ & $21.6^{b, c}$ & $24.6^{h, i}$ & $21.9^{b, d}$ & $26.8^{f, h}$ & $22.8^{b, e}$ & $28.2^{g, i}$ \\
\hline $\mathrm{SD}$ & 6.0 & 5.8 & 5.5 & 5.9 & 5.8 & 5.3 & 6.4 & 6.3 \\
\hline Minimum & 9.5 & 11.0 & 14.6 & 10.7 & 13.7 & 17.8 & 12.0 & 15.1 \\
\hline Maximum & 29.1 & 31.0 & 31.6 & 31.0 & 31.9 & 36.7 & 34.0 & 36.9 \\
\hline \multicolumn{9}{|c|}{ Minimum ulnar deviation } \\
\hline Average & $4.0^{b, c}$ & $0.8^{f, g}$ & $4.7^{b}$ & $1.8^{h, i}$ & 5.4 & $4.6^{f, h}$ & $6.4^{c}$ & $6.2^{g, i}$ \\
\hline
\end{tabular}

${ }^{a}$ Interactions between slope and hand existed for all 3 dependent variables $(P<.05)$. Statistical analysis consisted of a 2 -factor repeated-measures analysis of variance (ANOVA) ( $\mathrm{F}_{3,105}$ for interaction). Therefore, differences between hands are presented for each slope, and differences across slopes are presented individually for each hand.

${ }^{b}$ Indicates that the right wrist ulnar deviation angle was different $(P<.05)$ from the left wrist ulnar deviation for that slope. Statistical analysis consisted of a onefactor repeated-measures ANOVA ( $\mathrm{F}_{1,15}$ for hand main effect).

${ }^{c, d, e}$ Indicates that the left wrist ulnar deviation angle was different $(P<.05)$ from that of other slopes marked with same designation. Statistical analysis consisted of a 2-factor repeated-measures ANOVA ( $\mathrm{F}_{3,105}$ for slope main effect) and a Tukey honestly significant difference (HSD) multiple-comparison post hoc test.

$f, g, h, i$ Indicates that the right wrist ulnar deviation angle was different $(P<.05)$ from that of other slopes marked with same designation. Statistical analysis consisted of a 2-factor repeated-measures ANOVA ( $\mathrm{F}_{3,105}$ for slope main effect) and a Tukey HSD multiple-comparison post hoc test.

\section{Typing Performance}

There were no differences in typing speed and accuracy among the 4 slopes of the keyboard. As shown in Table 6 , mean typing speed ranged less than $1 \mathrm{wpm}$ across the slopes of the keyboards (range $=66.1-66.9 \mathrm{wpm}$ ). In addition, mean typing accuracy was $100 \%$ among the 4 slopes.

\section{Psychophysical Assessment of Ease of Use and Overall Comfort}

The keyboard at a slope of -15 degrees was rated less easy to use than the keyboard with slopes at $-7.5,0$, and 7.5 degrees. Likewise, the keyboard with a slope of -15 degrees was rated less comfortable than the keyboard with slopes at $-7.5,0$, and 7.5 degrees.

\section{Discussion}

\section{Wrist Position}

Wrist extension. Our results show that when the keyboard slope was changed by an amount of 22.5 degrees (from $7.5^{\circ}$ to $-15^{\circ}$ ), mean wrist extension decreased approximately 15 degrees (from $12^{\circ}$ of extension to $3^{\circ}$ of flexion). This represents a decrease of 2 degrees of wrist extension for each 3-degree downward change in slope of the keyboard. This finding is in general agreement with our earlier findings that wrist extension angle decreased as the keyboard's downward slope was increased. ${ }^{7}$ In our earlier study, ${ }^{7}$ mean wrist extension decreased approximately 15 degrees, from 23 degrees of wrist extension at the 15-degree slope to 8 degrees of wrist extension at the -15-degree slope. In that study, the ratio of wrist extension decrease to change in keyboard slope was 1:2. Mean wrist extension in the earlier study at the 7.5-degree slope was approximately 19 degrees, whereas subjects in our current study extended their wrists at a mean angle of 12.5 degrees for the same keyboard slope. We believe the difference in wrist extension between the 2 studies can be explained by the placement of the wrist rest in relationship to the keyboard. As shown in Figure 3, the wrist rest was integrated into the keyboard support tray in the present study, whereas in our previous study, ${ }^{7}$ the wrist rest was fixed horizontally regardless of keyboard slope. The wrist rest in the current study places the wrist in a similar position to that in a study by Hedge and Powers. ${ }^{25}$ They placed the wrist rest on the same plane as the keyboard. In the study by Hedge and Powers, the mean wrist extension angle was 1 degree of flexion for a -12-degree keyboard slope, which is comparable to the present study's angular results of 3 degrees of flexion at a -15-degree slope.

With a wrist rest in the same plane as the keyboard, the pivot point for combined keyboard and wrist rest struc- 


\section{Table 3.}

Mean, Standard Deviation, and Minimum and Maximum Values of 10th Percentile Amplitude Probability Distribution Function of Electromyographic Signals From the Extensor Carpi Ulnaris (ECU), Flexor Carpi Ulnaris (FCU), and Flexor Carpi Radialis (FCR) Muscles as Subjects Typed on a Computer Keyboard With Slopes at 4 Angles $^{a}$

\begin{tabular}{|c|c|c|c|c|c|c|c|c|}
\hline \multirow[b]{2}{*}{ Muscle } & \multicolumn{2}{|c|}{$7.5^{\circ}$ Slope } & \multicolumn{2}{|c|}{ 0 Slope } & \multicolumn{2}{|c|}{$-7.5^{\circ}$ Slope } & \multicolumn{2}{|c|}{$-15^{\circ}$ Slope } \\
\hline & Left & Right & Left & Right & Left & Right & Left & Right \\
\hline \multicolumn{9}{|l|}{$\operatorname{ECU}(n=15)^{e}$} \\
\hline SD & 3.0 & 3.7 & 2.6 & 3.0 & 2.4 & 3.1 & 2.1 & 2.8 \\
\hline Minimum & 3.2 & 2.0 & 3.5 & 1.1 & 2.4 & 0.6 & 3.0 & 0.1 \\
\hline Maximum & 14.7 & 16.8 & 12.8 & 12.8 & 11.8 & 12.5 & 10.1 & 10.7 \\
\hline $\bar{X}$ & 1.4 & 0.7 & 1.4 & 0.8 & 1.4 & 0.8 & 1.5 & 0.8 \\
\hline SD & 1.7 & 0.7 & 1.5 & 0.8 & 1.5 & 0.6 & 1.7 & 0.7 \\
\hline Minimum & 0.1 & 0.1 & 0.2 & 0.1 & 0.1 & 0.2 & 0.1 & 0.3 \\
\hline Maximum & 6.3 & 2.6 & 5.7 & 3.2 & 5.1 & 2.6 & 5.5 & 3.2 \\
\hline \multicolumn{9}{|l|}{ FCR $(n=16)$} \\
\hline $\bar{x}$ & 1.2 & 0.6 & 1.1 & 0.6 & 1.0 & 0.5 & 0.9 & 0.5 \\
\hline
\end{tabular}

${ }^{a}$ Units are in percentage of maximum voluntary contraction (MVC). No differences existed between right and left wrists.

${ }_{b, c, d}$ Average of left and right electromyographic percentage of MVC different from that of other slopes marked with same designation at the $P<.05$ level Statistical analysis consisted of one-factor analysis of variance $\left(\mathrm{F}_{3,42}\right.$ for slope main effect) and a Tukey honestly significant difference multiple-comparison post hoc test.

${ }^{e}$ Data for one subject had to be discarded for ECU muscle due to technical difficulties.

ture is near the user's wrist, as opposed to near the metacarpophalangeal joints when a horizontal, detached wrist rest is used with the keyboard. The location of the pivot point is important, because we believe that when the wrist rest is attached to the keyboard and follows the slope angle of the keyboard, the hands tend to follow the wrist rest, therefore resulting in less wrist extension than if the wrist rest remains horizontal. The difference in the orientation of the wrist rest to the keyboard may explain the approximately 12-degree difference of wrist extension we measured at the lowest negative slope relative to findings from our earlier study. ${ }^{7}$ In the present study, we found an average of 3 degrees of wrist flexion when the subjects were typing on a wrist rest in the same plane with the keyboard and with a slope of -15 degrees. Previously, we found an average of 9 degrees of wrist extension when the keyboard (and not the wrist rest) was positioned at a slope of -15 degrees. The design of the wrist rest on the same plane as the keyboard that we used in the present study appears to us to be more representative of typical computer workstations than use of a fixed horizontal wrist rest.

Based on carpal tunnel pressure studies, wrist extension angles closer to neutral are believed to be beneficial with respect to etiology of nerve conduction injuries affecting the wrist. ${ }^{8,10}$ With wrist extension angles greater than 15 degrees, pressure in the carpal tunnel could result in more pressure against the median nerve, and this could contribute to the development or perpetuation of carpal tunnel syndrome. Therefore, typing with a wrist position close to an anatomically neutral position could minimize pressure in the carpal tunnel, and this theoretically could benefit individuals with a diagnosis of carpal tunnel syndrome. Data from our study show that mean wrist extension angles for all positions of the keyboard (slopes of $7.5^{\circ}$ to $-15^{\circ}$ ) were 15 degrees or less. However, maximum wrist extension angles exceeded 15 degrees and approached 25 degrees at the 7.5-degree slope. Thus, keyboards positioned at a slope of 7.5 degrees may increase pressure in the carpal tunnel because, based on our data, the wrist is often extended beyond 15 degrees from neutral during the normal activity of typing.

Wrist extension angles closer to a neutral position, theoretically, could decrease the probability of developing other MSDs affecting the hand and wrist. Modeling of the tendons in the wrist with a free-body diagram showed that the reaction force exerted against the tendons from the carpal bones and flexor retinaculum increased as the wrist was extended.26,27 Dynamics applied to the static model showed that acceleration of the wrist in the flexion/extension plane increased the reaction forces on the tendons even more than in the static analysis. ${ }^{9}$ Greater reaction forces against the tendons and their sheaths that pass through the wrist, theoretically, could increase the risk of tendinitis or tenosynovitis. 


\section{Table 4.}

Mean, Standard Deviation, and Minimum and Maximum Values of 50th Percentile Amplitude Probability Distribution Function of Electromyographic Signals From the Extensor Carpi Ulnaris (ECU), Flexor Carpi Ulnaris (FCU), and Flexor Carpi Radialis (FCR) Muscles as Subjects Typed on a Computer Keyboard With Slopes at 4 Angles ${ }^{a}$

\begin{tabular}{|c|c|c|c|c|c|c|c|c|}
\hline Muscle & \multicolumn{2}{|c|}{$7.5^{\circ}$ Slope } & \multicolumn{2}{|c|}{$0^{\circ}$ Slope } & \multicolumn{2}{|c|}{$-7.5^{\circ}$ Slope } & \multicolumn{2}{|c|}{$-15^{\circ}$ Slope } \\
\hline \multicolumn{9}{|l|}{$\operatorname{ECU}(n=15)^{e}$} \\
\hline SD & 5.6 & 6.1 & 4.9 & 4.9 & 4.8 & 5.0 & 4.0 & 4.5 \\
\hline Minimum & 6.3 & 6.0 & 6.7 & 5.8 & 5.6 & 5.0 & 6.0 & 4.4 \\
\hline Maximum & 26.9 & 30.0 & 23.3 & 24.4 & 22.1 & 24.2 & 19.8 & 21.4 \\
\hline $\bar{x}$ & $7.0^{b}$ & 4.2 & 7.0 & 4.7 & 7.6 & 5.0 & $8.0^{b}$ & 5.3 \\
\hline SD & 5.2 & 1.8 & 4.6 & 2.0 & 5.1 & 2.2 & 5.5 & 2.4 \\
\hline Minimum & 1.2 & 1.1 & 1.9 & 1.4 & 1.6 & 1.2 & 1.6 & 1.1 \\
\hline Maximum & 18.2 & 7.8 & 17.7 & 8.7 & 17.5 & 9.5 & 19.1 & 11.2 \\
\hline \multicolumn{9}{|l|}{ FCR $(n=16)^{f}$} \\
\hline $\bar{X}$ & 3.7 & 1.8 & 3.6 & 2.0 & 3.8 & 1.9 & 3.6 & 1.9 \\
\hline
\end{tabular}

${ }^{a}$ Units are in percentage of maximum voluntary contraction (MVC).

${ }_{b, c, d}$ Average of left and right electromyographic percentages of MVC different from that of other slopes marked with same designation at the $P<.05$ level. Statistical analysis consisted of one-factor analysis of variance (ANOVA) ( $F_{3,41}$ for slope main effect) and a Tukey honestly significant difference multiplecomparison post hoc test.

${ }^{e}$ Data for one subject had to be discarded for ECU muscle due to technical difficulties.

${ }^{f}$ Right side different from left side for FCR and FCU muscles $(P<.05)$. Statistical analysis consisted of a 2 -factor ANOVA $\left(\mathrm{F}_{1,105}\right.$ for hand main effect).

No difference existed between right and left wrists for ECU muscle.

Ulnar deviation. The ulnar deviation angles of approximately 13 and 9 degrees for the left and right wrists for the 7.5-degree slope, which are similar to the built-in slopes of many commercially available keyboards, are similar to ulnar deviation angles measured on 90 subjects who typed on a conventional keyboard in a previous study. ${ }^{7}$ As we found in our earlier study with keyboards positioned at negative slopes, ulnar deviation tended to increase 3 to 5 degrees when the keyboard slope was changed from 7.5 degrees to -15 degrees. ${ }^{7}$ The reason for this increase in ulnar deviation is not clear at this time, but a change in forearm pronation (which was not measured in this study) could play a role. Typically, typists pronate their forearms approximately 65 degrees when they type on a conventional keyboard, ${ }^{4}$ which means the wrists are not parallel to the keyboard surface. We found that subjects typing on tilted (also called "tented" or "vertically inclined") keyboards reduced forearm pronation by approximately 20 to 25 degrees compared with subjects using a conventional keyboard, and they reduced ulnar deviation by approximately 12 to 14 degrees in the left and right wrists, respectively. ${ }^{3}$ Giving the keyboard a downward slope may have an effect of increasing forearm pronation and thereby increasing ulnar deviation at the wrist. Because we did not measure forearm pronation, we cannot determine whether this hypothesis is correct. Regardless of the reason for an increase in ulnar deviation, the increase may partially counteract any benefits of positioning a keyboard with a downward slope because increased ulnar deviation, in theory, increases the net reaction forces and friction on the tendons passing through the wrist. ${ }^{26,27}$

\section{Forearm Muscle EMG Activity}

Surface EMG activity, calibration, and APDF. Although more than a score of relatively small muscles pass through the forearm and thus present opportunities for cross talk during EMG recording, researchers have shown that surface EMG is a reliable and accurate method to assess electrical activity of the ECU, FCR, and FCU muscles. ${ }^{15-17}$ In our study, the EMG activity of these muscles was measured for normalization with the wrists extended 8 degrees and ulnarly deviated 10 degrees on the right wrist and 15 degrees on the left wrist, which is the average of wrist positions required for typing on conventional keyboards. ${ }^{4}$ Therefore, errors in EMG measurement due to change in length of muscles, in our opinion, were minimized.

The APDF method of analyzing EMG data recorded during an activity where a limb moves has been used often in investigations of upper-extremity muscle activity. $5,6,28$ The APDF model, which was described and illustrated by Jonsson, ${ }^{29}$ states that the static level of muscle activity is the percentage of MVC that is below the lowest $10 \%$ of the cumulative RMS EMG signal, 


\section{Table 5.}

Mean, Standard Deviation, and Minimum and Maximum Values of 90th Percentile Amplitude Probability Distribution Function of Electromyographic Signals From the Extensor Carpi Ulnaris (ECU), Flexor Carpi Ulnaris (FCU), and Flexor Carpi Radialis (FCR) Muscles as Subjects Typed on a Computer Keyboard With Slopes at 4 Angles $^{a}$

\begin{tabular}{|c|c|c|c|c|c|c|c|c|}
\hline Muscle & \multicolumn{2}{|c|}{$7.5^{\circ}$ Slope } & \multicolumn{2}{|c|}{$0^{\circ}$ Slope } & \multicolumn{2}{|c|}{$-7.5^{\circ}$ Slope } & \multicolumn{2}{|c|}{$-15^{\circ}$ Slope } \\
\hline \multicolumn{9}{|l|}{$\operatorname{ECU}(n=15)^{d}$} \\
\hline SD & 11.5 & 11.0 & 10.1 & 9.0 & 10.2 & 10.4 & 8.6 & 8.7 \\
\hline Minimum & 11.4 & 11.7 & 11.3 & 14.5 & 10.1 & 12.8 & 11.6 & 12.7 \\
\hline Maximum & 55.7 & 57.2 & 47.7 & 48.0 & 46.8 & 52.2 & 43.1 & 43.8 \\
\hline $\bar{x}$ & $27.1^{b}$ & 18.8 & $26.5^{c}$ & 20.0 & 27.8 & 21.3 & $28.5^{b, c}$ & 22.4 \\
\hline SD & 17.3 & 10.1 & 14.9 & 10.6 & 15.8 & 11.9 & 16.3 & 12.0 \\
\hline Minimum & 11.0 & 7.1 & 12.6 & 7.9 & 12.7 & 8.9 & 11.3 & 6.0 \\
\hline Maximum & 65.4 & 49.6 & 65.3 & 51.3 & 68.4 & 59.1 & 70.7 & 58.6 \\
\hline \multicolumn{9}{|l|}{$\operatorname{FCR}(n=16)^{e}$} \\
\hline $\bar{x}$ & 13.5 & 9.3 & 13.5 & 9.7 & 13.9 & 9.7 & 13.6 & 9.7 \\
\hline
\end{tabular}

${ }^{a}$ Units are in percentage of maximum voluntary contraction (MVC).

${ }^{b, c}$ Average of left and right electromyographic percentage of MVC different from that of other slopes marked with same designation at the $P<.05$ level. Statistical analysis consisted of one-factor analysis of variance (ANOVA) ( $\mathrm{F}_{3,41}$ for slope main effect) and a Tukey honestly significant difference multiple-comparison post hoc test.

${ }^{d}$ Data for one subject had to be discarded for ECU muscle due to technical difficulties.

${ }^{e}$ Right side different from left side for FCU and FCR muscles $(P<.05)$. Statistical analysis consisted of 2 -factor ANOVA $\left(\mathrm{F}_{1,105}\right.$ for hand main effect). No difference existed between right and left wrists for ECU muscle.

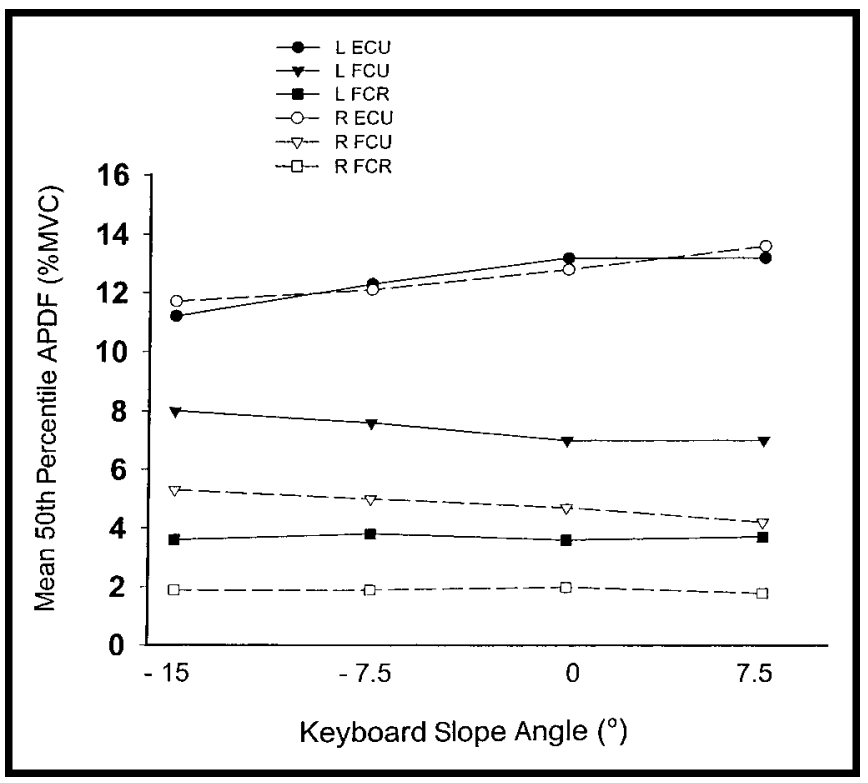

Figure 7.

Mean 50 th percentile amplitude probability distribution function (APDF) of the forearm muscles as a function of keyboard slope angle and hand. The muscles are extensor carpi ulnaris (ECU), flexor carpi ulnaris (FCU), and flexor carpi radialis (FCR). Units are in percentage of maximum voluntary contraction (\%MVC). whereas the dynamic level (90th percentile APDF) is the percentage of MVC that is above the greatest $10 \%$ of the cumulative EMG signal.

Magnitude of EMG activity. The percentages of MVC of the 10th, 50th, and 90th percentile APDFs for the ECU muscle from this study $(6 \%, 12 \%$, and $24 \%$, respectively) are similar to results reported by Gerard et al, ${ }^{5}$ who found percentages of $6 \%, 11 \%$, and $19 \%$ MVC for APDFs of the finger extensor muscles. Fernstrom et $\mathrm{al}^{6}$ measured a range of $7 \%$ to $12 \%$ MVC for the 50th percentile APDF for the extensor digitorum muscle while subjects typed on computer keyboards and typewriters.

As for the ECU muscle, the percentages of MVC for the wrist flexors (FCU and FCR muscles) in our study are comparable to those reported in the literature..$^{5,8}$ Although Gerard et $\mathrm{al}^{5}$ measured EMG activity from the finger flexors (and not the wrist flexors [ie, FCR and FCU muscles] as in our present study), their results show similar percentage of MVC. Measurements of approximately $2 \%, 6 \%$, and $19 \%$ MVC for the 10th, 50th, and 90th percentile APDFs from the finger flexor muscles ${ }^{8}$ are in agreement with our findings of approximately $1 \%$, $4 \%$, and $10 \%$ MVC for the FCR muscle and 1\%, $10 \%$, and 28\% MVC for the FCU muscle for the 10th, 50th, and 90th APDFs, respectively. 


\section{Table 6.}

Mean, Standard Deviation, and Minimum and Maximum Values of Typing Speed (in Words per Minute) and Accuracy From Typing on a Keyboard With Slopes at 4 Angles $^{a}$

\begin{tabular}{|c|c|c|c|c|}
\hline & $\begin{array}{l}7.5^{\circ} \\
\text { Slope }\end{array}$ & $\begin{array}{l}0^{\circ} \\
\text { Slope }\end{array}$ & $\begin{array}{l}-7.5^{\circ} \\
\text { Slope }\end{array}$ & $\begin{array}{l}-15^{\circ} \\
\text { Slope }\end{array}$ \\
\hline \multicolumn{5}{|l|}{ Typing speed (wpm) } \\
\hline $\bar{x}$ & 66.1 & 66.9 & 66.9 & 66.5 \\
\hline SD & 13.7 & 15.2 & 15.8 & 14.5 \\
\hline Minimum & 45 & 40 & 42 & 40 \\
\hline Maximum & 92 & 92 & 97 & 91 \\
\hline Typing accuracy (\%) & 100 & 100 & 100 & 100 \\
\hline
\end{tabular}

${ }^{a}$ No differences were found among the 4 keyboard slope angles for either variable.

Overall, the percentage of MVC was greater for the ECU muscle than for the wrist flexors. The percentage of MVC for the ECU muscle was twice as large as the percentage of MVC for the FCR and FCU muscles for the 10th and 50th percentile APDFs. The role of the ECU muscle in typing is to hold the wrists in an extended and ulnarly deviated position above the keyboard so a user can minimize the distance required to reach the keys. The role of the wrist flexors in a typing task is to move the wrist in the radial/ulnar plane to type alphabetic, numeric, and special function keys that are located toward the perimeter of the keyboard. The percentage of MVC was greater for the FCU muscle than for the FCR muscle at the 50th and 90th percentile APDFs, but not at the 10th percentile APDF. Although the 10th percentile APDF for both the FCR and FCU muscles averaged about $1 \%$ MVC, the percentage of MVC was $3 \%$ greater for the FCU muscle than for the FCR muscle at the 50th percentile APDF and about $10 \%$ greater for the 90th percentile APDF. The greater EMG activity of the FCU muscle over the FCR muscle is apparently due to the ulnarly deviated wrist position required when typing. The FCU muscle is used to ulnarly deviate the wrist approximately 10 degrees or more to type on a conventional keyboard ${ }^{4}$ and also to ulnarly deviate the wrist to type numeric and special function keys that are located to the ulnar side of both wrists. We found in our present study and in our earlier work ${ }^{3,4,24}$ that the left wrist is ulnarly deviated up to 5 degrees more than the right wrist while typing on conventional and split keyboards. This finding may explain why the percentage of MVC for the FCU muscle was greater for the left wrist than for the right wrist (approximately 2\% MVC greater for 50th percentile APDF, as illustrated in Fig. 7). In theory, the FCU muscle is required to exert more tension to ulnarly deviate the wrist 5 degrees more than the right wrist. Whether a small difference of $2 \%$ MVC of the FCU muscle is clinically relevant is not known.

Effect of keyboard slope on forearm EMG activity. As the keyboard slopes increased in a downward direction from a positive angle of 7.5 degrees to a negative angle of 15 degrees, the percentage of MVC for the ECU muscle decreased $2 \%$ to $3 \% \mathrm{MVC}$ across all percentile ranges of APDF. Based on the assumption that percentage of MVC is representative of muscle tension, this decrease in percentage of MVC for the ECU muscle indicates that less EMG activity of the ECU muscle is needed when typing on a negatively sloped keyboard. This simple interpretation is tempered by the dynamic nature of the ECU muscle during typing and by the fact that wrist angle (and therefore muscle length) also changed as the keyboard was adjusted, as did the moment arm of the muscle. The clinical significance of a $2 \%$ or $3 \%$ change in percentage of MVC for the ECU muscle is difficult to interpret. Although a $2 \%$ to $3 \%$ difference is seemingly very small, and likely of no clinical importance when typing for short periods of time, we believe that such a difference may be important when sustained for several hours per day.

The results of our study show that positioning the keyboard with a downward slope did not lead to an increase in the muscle activity of the wrist extensors. Furthermore, the overall decrease of the percentage of MVC for the ECU muscle as the keyboard was positioned with a downward slope provides some insight that warrants future study. If future research indicates that there is a clinically relevant decrease in ECU muscle EMG activity with the keyboard positioned with a downward slope, then this keyboard positioning may reduce muscle activity in the forearm muscles. This could reduce the pain and discomfort that some people experience while typing.

Although the magnitude of RMS EMG activity has been shown to increase with muscle fatigue over long durations of contraction, ${ }^{30}$ fatigue did not likely come into play in our study. The duration of typing trials were short enough (6 minutes), in our view, to minimize effects from fatigue and therefore any confounding effect on EMG activity levels. In addition, random assignment of the slopes of the keyboard should have eliminated any systematic bias during testing.

There was an increase of $1 \%$ to $1.5 \%$ MVC in the muscle activity of the FCU muscle as the keyboard downward slope increased. This change could be related to the increased ulnar deviation noted with a keyboard with a negative slope. Again, although these changes are small, they may warrant consideration in the design of keyboards. The incorporation of a slant angle-where the keyboard is split into halves and the halves are angled outward-in the design of keyboards with negative slopes would be justified because the slant angle incorporated in split keyboards tends to place the wrists in a relatively neutral anatomical posture. ${ }^{3}$ 


\section{Typing Performance}

The narrow ranges of mean typing speeds (66.1-66.9 wpm) and $100 \%$ accuracy for all the keyboard slopes indicate to us that subjects quickly adapted to new keyboard slope angles, even after only 3 minutes of practice. The typing performance we found is similar to results from our previous study, ${ }^{7}$ where we showed that keyboards with negative slopes did not impair typing performance. We expected perfect typing accuracy because subjects were allowed to correct errors as they typed.

\section{Ease of Use}

The assessments of ease of use and comfort show that the keyboard with a slope of -15 degrees was rated least comfortable and most difficult to use of all the keyboards tested.

\section{Future Work}

Our studies, including the current study, on keyboards with negative slopes were conducted in the laboratory and thus may not be representative of what actually occurs with wrist positions in real-world office settings.

\section{Conclusion}

Deviated wrist posture in the flexion/extension plane is thought to be a risk factor for MSDs affecting the hand and wrist. Changing the slope of a computer keyboard downward from its typical built-in slope angle of approximately 6 degrees produced the following results: reduction in wrist extension angle to near neutral position, a slight decrease in EMG activity of a major wrist extensor (ECU muscle), and no impairment of typing performance for 10-digit "touch" typists. The effect of less EMG activity of the ECU muscle with a downward-sloping keyboard remains to be established. With a keyboard at a downward slope, there was also an increase in ulnar deviation of the wrists. This increased ulnar deviation may counteract the effect from decreased wrist extension.

Whether positioning a keyboard downward from its built-in slope is helpful in preventing or managing distal upper-extremity MSDs attributed to the use of keyboards is not known and warrants further study. However, data on wrist extension from our study considered in light of the theoretical biomechanical modeling of the wrist suggest that a keyboard with a neutral (horizontal) slope or a keyboard with a downward slope might have beneficial effects to prevent or treat upper-extremity injuries related to the frequent use of computer keyboards.

\section{References}

1 Tittiranonda P, Burastero S, Rempel D. Risk factors for musculoskeletal disorders among computer users. Occupational Medicine: State of the Art Reviews. 1999;14:17-38.
2 Kroemer KHE. Human engineering the keyboard. Human Factors. 1972;14:51-63.

3 Marklin RW, Simoneau GG, Monroe JF. Wrist and forearm posture from typing on split and vertically-inclined computer keyboards. Human Factors. 1999;41:559-569.

4 Simoneau GG, Marklin RW, Monroe JF. Wrist and forearm posture of users of conventional computer keyboards. Human Factors. 1999;41: $413-424$

5 Gerard MJ, Armstrong TJ, Franzblau A, et al. The effects of keyswitch stiffness on typing force, finger electromyography, and subjective discomfort. Am Ind Hyg Assoc J. 1999;60:762-769.

6 Fernstrom E, Ericson MO, Malker H. Electromyographic activity during typewriter and keyboard use. Ergonomics. 1994;37:477-484.

7 Simoneau GG, Marklin RW. Effect of computer keyboard slope and height on wrist extension angle. Human Factors. 2001;43:287-298.

8 Rempel D, Kier PJ, Smutz WP, Hargen A. Effects of static fingertip loading on carpal tunnel pressure. J Orthop Res. 1997;15:422-426.

9 Schoenmarklin RW, Marras WS. A dynamic biomechanical model of the wrist joint. In: Proceedings of the 34th Annual Meeting of the Human Factors Society. Orlando, Fla: Human Factors and Ergonomics Society; 1990:805-809.

10 Kier PJ, Bach JM, Rempel DM. Effects of finger posture on carpal tunnel pressure during wrist motion. J Hand Surg [Am]. 1998;23: 1004-1009.

11 Lieber RL, Friden J. Musculoskeletal balance of the human wrist elucidated using intraoperative laser diffraction. J Electromyogr Kinesiol. 1998;8:93-100.

12 Loren GJ, Shoemaker SD, Burkholder TJ, et al. Human wrist motors: biomechanical design and application to tendon transfers. J Biomech. 1996;29:331-342.

13 Durkan JA. A new diagnostic test for carpal tunnel syndrome.J Bone Joint Surg Am. 1991;73:535-538.

14 Gellman H, Gelberman RH, Tan AM, Botte MJ. Carpal tunnel syndrome: an evaluation of the provocative diagnostic tests. J Bone Joint Surg Am. 1986;68:735-737.

15 Martin B, Rempel D, Sudarsan P, et al. Accuracy of surface EMG to assess forearm muscle load during typing. In: Proceedings of the Marconi Research Conference; Marshall, Calif; December 11-14, 1998. Marshall, Calif: Marconi Conference Center; 1998:55-60.

16 Martin B, Rempel D, Sudarsan P, et al. Reliability and sensitivity of methods to quantify muscle load during keyboard work. In: Proceedings of the Marconi Research Conference; Marshall, Calif; December 1997. Marshall, Calif: Marconi Conference Center; 1997:40-42.

17 Jacobson M, Rempel D, Martin B, et al. Comparison of fine wire to surface EMG amplitude of finger muscles during precision tasks. In: Proceedings of the Marconi Research Conference; Marshall, Calif; December 11-14, 1998. Marshall, Calif: Marconi Conference Center; 1998:49-54.

18 Portney LG, Watkins MP. Foundations of Clinical Research: Applications to Practice. 2nd ed. Upper Saddle River, NJ: Prentice-Hall Health; 2000.

19 Shrout PE, Fleiss J. Intraclass correlations: uses in assessing rate reliability. Psychol Bull. 1979;86:420-428.

20 Soderberg G, Blanco M, Cosentino T, Kurdelmeier K. An EMG analysis of posterior trunk musculature during flat and anteriorly inclined sitting. Human Factors. 1986;28:483-491.

21 Neumann D, Cook T, Sholty R, Sobush D. An electromyographic analysis of hip abductor muscle activity when subjects are carrying loads in one or both hands. Phys Ther. 1992;72:207-217. 
22 Basmajian JV, Blumenstein R. Electrode Placement in EMG Biofeedback. Baltimore, Md: Williams \& Wilkins; 1980.

23 American National Standard for Human Factors Engineering of Visual Display Workstations. Santa Monica, Calif: Human Factors Society Inc; 1988. American National Standards Institute/Human Factors Society Standard No. 1001988.

24 Marklin RW, Simoneau GG. Effect of setup configurations of split computer keyboards on wrist angle. Phys Ther. 2001;81;1038-1048.

25 Hedge A, Powers JR. Wrist postures while keyboarding: effects of a negative slope keyboard system and full motion forearm supports. Ergonomics. 1995;38:508-517.

26 Armstrong TJ, Chaffin DB. Some biomechanical aspects of the carpal tunnel. J Biomech. 1978;12:567-570.
27 Armstrong TJ, Chaffin DB. An investigation of the relationship between displacements of the finger and wrist joints and the extrinsic finger flexor tendons. J Biomech. 1978;11:119-128.

28 Strasser H, Keller E, Muller KW, Ernst J. Local muscular strain dependent on the direction of horizontal arm movements. Ergonomics. 1989;32:899-910.

29 Jonsson B. Measurement and evaluation of local muscular strain in the shoulder during constrained work. Journal of Human Ergology. $1982 ; 11 ; 73-88$.

30 Jensen BR, Laursen B, Sjogaard G. Aspects of shoulder function in relation to exposure demands and fatigue: a mini review. Clin Biomech. 2000;15(suppl 1):S17-S20. 